\title{
Low Complexity Region
}

National Cancer Institute

\section{Source}

National Cancer Institute. Low Complexity Region. NCI Thesaurus. Code C49032.

A protein region consisting of a very small variety of residues, including homopolymeric

runs, short-period repeats, and more subtle overrepresentation of one or a few residues.

(from Biolnformatics Glossary: big.mcw.edu) 